\title{
Parameter Dependence of Positive Solutions for Second-Order Singular Neumann Boundary Value Problems with Impulsive Effects
}

\author{
Xuemei Zhang \\ Department of Mathematics and Physics, North China Electric Power University, Beijing 102206, China \\ Correspondence should be addressed to Xuemei Zhang; zxm74@sina.com
}

Received 21 March 2014; Accepted 7 May 2014; Published 19 May 2014

Academic Editor: Juan J. Nieto

Copyright (c) 2014 Xuemei Zhang. This is an open access article distributed under the Creative Commons Attribution License, which permits unrestricted use, distribution, and reproduction in any medium, provided the original work is properly cited.

The author considers the Neumann boundary value problem $-y^{\prime \prime}(t)+\mathbf{M} y(t)=\lambda \omega(t) f(t, y(t)), t \in J, t \neq t_{k},-\left.\Delta y^{\prime}\right|_{t=t_{k}}=$ $\lambda I_{k}\left(t_{k}, y\left(t_{k}\right)\right), k=1,2, \ldots, m, y^{\prime}(0)=y^{\prime}(1)=0$ and establishes the dependence results of the solution on the parameter $\lambda$, which cover equations without impulsive effects and are compared with some recent results by Nieto and O'Regan.

\section{Introduction}

Impulsive effects exist widely in many evolution processes in which their states are changed abruptly at certain moments of time. The theory and applications of impulsive differential equations have been emerging as an important area of investigation in recent years [1-6]. There is a vast literature on the existence of solutions by using different methods such as bifurcation theory $[7,8]$, fixed point theorems in cones [9-12], the method of lower and upper solutions [13, $14]$, and the theory of critical point theory and variational methods [15-19]. We remark that on second-order impulsive differential equations with parameter only a few results have been obtained; see, for instance, $[9,20-22]$. To the best of our knowledge, these papers only consider the existence of positive solutions. However, the corresponding results for the dependence of the solution on the parameter $\lambda$ for secondorder impulsive differential equations are not investigated until now. In this paper, we try to solve this kind of problem.

Consider the Neumann boundary value problems

$$
\begin{array}{r}
-y^{\prime \prime}(t)+\mathbf{M} y(t)=\lambda \omega(t) f(t, y(t)), \\
t \in J, \quad t \neq t_{k},
\end{array}
$$

$$
\begin{gathered}
-\left.\Delta y^{\prime}\right|_{t=t_{k}}=\lambda I_{k}\left(t_{k}, y\left(t_{k}\right)\right), \quad k=1,2, \ldots, m, \\
y^{\prime}(0)=y^{\prime}(1)=0,
\end{gathered}
$$

where $\mathbf{M}>0$ is a constant, $\lambda>0$ is a parameter, $J=[0,1], \omega$ is a nonnegative measurable function on $(0,1), \omega \neq 0$ on any open subinterval in $(0,1)$, which may be singular at $t=0$ and/or $t=1, t_{k}(k=1,2, \ldots, m)$ (where $m$ is fixed positive integer) are fixed points with $0=t_{0}<t_{1}<t_{2}<\cdots<t_{k}<$ $\cdots<t_{m}<t_{m+1}=1$, and $\left.\Delta y^{\prime}\right|_{t=t_{k}}=y^{\prime}\left(t_{k}^{+}\right)-x^{\prime}\left(t_{k}^{-}\right)$, where $y^{\prime}\left(t_{k}^{+}\right)$and $y^{\prime}\left(t_{k}^{-}\right)$represent the right-hand limit and left-hand limit of $y^{\prime}(t)$ at $t=t_{k}$, respectively. In addition, $\omega, f$, and $I_{k}$ satisfy

$$
\begin{aligned}
& \left(\mathrm{H}_{1}\right) \omega \in L_{\mathrm{loc}}^{1}(0,1) \text {; } \\
& \left(\mathrm{H}_{2}\right) f \in C\left(J \times \mathscr{R}^{+}, \mathscr{R}^{+}\right), I_{k} \in C\left(J \times \mathscr{R}^{+}, \mathscr{R}^{+}\right) \text {, where } \\
& \mathscr{R}^{+}=[0,+\infty), k=1,2, \ldots, m \text {. }
\end{aligned}
$$

Some special cases of (1) have been investigated. For example, Nieto and O'Regan [17] studied problem (1) with $\lambda=1$ and $\omega \equiv 1$ for $t \in J$. By using variational methods and critical point theory, the authors proved the existence of solutions of problem (1). 
For ease of exposition, we set

$$
\begin{array}{rl}
f^{0} & =\limsup _{y \rightarrow 0^{+}} \max _{t \in J} \frac{f(t, y)}{y}, \\
f^{\infty} & =\limsup _{y \rightarrow \infty} \max _{t \in J} \frac{f(t, y)}{y}, \\
f_{0} & =\liminf _{y \rightarrow 0^{+}} \min _{t \in J} \frac{f(t, y)}{y}, \\
f_{\infty} & =\liminf _{y \rightarrow \infty} \min _{t \in J} \frac{f(t, y)}{y}, \\
I^{0}(k) & =\limsup _{y \rightarrow 0^{+}} \max _{t \in J} \frac{I_{k}(t, y)}{y}, \\
I^{\infty}(k) & =\limsup _{y \rightarrow \infty} \max _{t \in J} \frac{I_{k}(t, y)}{y}, \\
I_{0}(k) & =\liminf _{y \rightarrow 0^{+}} \min _{t \in J} \frac{I_{k}(t, y)}{y}, \\
I_{\infty}(k) & =\liminf _{y \rightarrow \infty} \min _{t \in J} \frac{I_{k}(t, y)}{y}, \\
k & 1,2, \ldots, m .
\end{array}
$$

Our main results are as follows.

Theorem 1. Assume that $\left(H_{1}\right)$ and $\left(H_{2}\right)$ hold. Then the following two conclusions hold:

$\left(\mathrm{H}_{3}\right)$ if $f^{0}=0, I^{0}(k)=0$, and $f_{\infty}=\infty, I_{\infty}(k)=\infty$, $k=1,2, \ldots, m$, then for every $\lambda>0$ problem (1) has a positive solution $y_{\lambda}(t)$ satisfying $\lim _{\lambda \rightarrow 0^{+}}\left\|y_{\lambda}\right\|_{P C^{1}}=$ $\infty$;

$\left(\mathrm{H}_{4}\right)$ if $f_{0}=\infty, I_{0}(k)=\infty$, and $f^{\infty}=0, I^{\infty}(k)=0$, $k=1,2, \ldots, m$, then for every $\lambda>0$ problem (1) has a positive solution $y_{\lambda}(t)$ satisfying $\lim _{\lambda \rightarrow 0^{+}}\left\|y_{\lambda}\right\|_{P C^{1}}=0$.

Remark 2. Assume that $\left(\mathrm{H}_{1}\right)$ and $\left(\mathrm{H}_{2}\right)$ hold. Furthermore, suppose that $f_{\infty}=\infty$ or $I_{\infty}(k)=\infty, k=1,2, \ldots, m$, in $\left(\mathrm{H}_{3}\right)$ or $f_{0}=\infty$ or $I_{0}(k)=\infty, k=1,2, \ldots, m$, in $\left(\mathrm{H}_{4}\right)$. Then the conclusions of Theorem 1 also hold.

Remark 3. It follows from the conditions of Theorem 1 that we develop some ideas of Guo and Lakshmikantham essentially; for detail, see Theorem 2.3.7 in [23].

Remark 4. For simplicity we only consider Neumann boundary conditions since all the results obtained in this paper can also be adapted with minor changes to the other boundary conditions.

\section{Preliminaries}

Let $J^{\prime}=J \backslash\left\{t_{1}, t_{2}, \ldots, t_{m}\right\}, k=1,2, \ldots, m$, and

$$
\begin{gathered}
\mathrm{PC}^{1}[0,1]=\left\{y \in C[0,1]: y_{\left(t_{k}, t_{k+1}\right)} \in C\left(t_{k}, t_{k+1}\right),\right. \\
y^{\prime}\left(t_{k}^{-}\right), y^{\prime}\left(t_{k}^{+}\right) \text {exists, } \\
k=1,2, \ldots, m\} .
\end{gathered}
$$

Then $\mathrm{PC}^{1}[0,1]$ is a real Banach space with norm

$$
\|y\|_{\mathrm{PC}^{1}}=\max \left\{\|y\|_{\infty},\left\|y^{\prime}\right\|_{\infty}\right\},
$$

where $\|y\|_{\infty}=\sup _{t \in J}|y(t)|,\left\|y^{\prime}\right\|_{\infty}=\sup _{t \in J}\left|y^{\prime}(t)\right|$.

A function $y \in \operatorname{PC}^{1}[0,1] \cap C^{2}\left(J^{\prime}\right)$ is called a solution of problem (1) if it satisfies (1).

In our main results, we will make use of the following lemmas.

Lemma 5. If $\left(H_{1}\right)$ and $\left(H_{2}\right)$ hold, then problem (1) has a unique solution $y$ given by

$$
\begin{aligned}
y(t)= & \lambda \int_{0}^{1} G(t, s) \omega(s) f(s, y(s)) d s \\
& +\lambda \sum_{k=1}^{m} G\left(t, t_{k}\right) I_{k}\left(t_{k}, y\left(t_{k}\right)\right),
\end{aligned}
$$

where

$$
G(t, s)= \begin{cases}\frac{\cosh (\sqrt{\mathbf{M}}(1-t)) \cosh (\sqrt{\mathbf{M}} s)}{\sqrt{\mathbf{M}} \sinh (\sqrt{\mathbf{M}})}, & 0 \leq s \leq t \leq 1, \\ \frac{\cosh (\sqrt{\mathbf{M}} t) \cosh (\sqrt{\mathbf{M}}(1-s))}{\sqrt{\mathbf{M}} \sinh (\sqrt{\mathbf{M}})}, & 0 \leq t \leq s \leq 1 .\end{cases}
$$

Proof. The proof is similar to that of Lemma 2.4 in [24].

By (6), we can prove that $G(t, s)$ has the following property:

$$
\begin{array}{r}
\frac{1}{\sqrt{\mathbf{M}} \sinh (\sqrt{\mathbf{M}})}=\alpha \leq G(t, s) \leq \beta=\frac{\cosh ^{2}(\sqrt{\mathbf{M}})}{\sqrt{\mathbf{M}} \sinh (\sqrt{\mathbf{M}})}, \\
\forall t, s \in J .
\end{array}
$$

Define a cone in $\operatorname{PC}^{1}[0,1]$ by

$$
K=\left\{y \in \operatorname{PC}^{1}[0,1]: y \geq 0, y(t) \geq \delta\|y\|_{\mathrm{PC}^{1}}, t \in J\right\},
$$

where

$$
\delta=\frac{1}{\cosh ^{2}(\sqrt{\mathbf{M}})} .
$$

It is easy to see $K$ is a closed convex cone of $\mathrm{PC}^{1}[0,1]$. 
Define an operator $T_{\lambda}: K \rightarrow \operatorname{PC}^{1}[0,1]$ by

$$
\begin{aligned}
\left(T_{\lambda} y\right)(t)= & \lambda \int_{0}^{1} G(t, s) \omega(s) f(s, y(s)) d s \\
& +\lambda \sum_{k=1}^{m} G\left(t, t_{k}\right) I_{k}\left(t_{k}, y\left(t_{k}\right)\right) .
\end{aligned}
$$

From (10), we know that $y \in \operatorname{PC}^{1}[0,1]$ is a solution of problem (1) if and only if $y$ is a fixed point of operator $T_{\lambda}$.

Lemma 6. Suppose that $\left(H_{1}\right)$ and $\left(H_{2}\right)$ hold. Then $T_{\lambda}(K) \subset K$ and $T_{\lambda}: K \rightarrow K$ is completely continuous.

Proof. For $y \in K$, it follows from (7) and (10) that

$$
\begin{aligned}
(T y)(t)= & \lambda \int_{0}^{1} G(t, s) \omega(s) f(s, y(s)) d s \\
& +\lambda \sum_{k=1}^{m} G\left(t, t_{k}\right) I_{k}\left(t_{k}, y\left(t_{k}\right)\right) \\
\leq & \lambda \frac{\cosh ^{2}(\sqrt{\mathbf{M}})}{\sqrt{\mathbf{M}} \sinh (\sqrt{\mathbf{M}})} \\
& \times\left[\int_{0}^{1} \omega(s) f(s, y(s)) d s+\sum_{k=1}^{m} I_{k}\left(t_{k}, y\left(t_{k}\right)\right)\right]
\end{aligned}
$$

It follows from (7), (10), and (11) that

$$
\begin{aligned}
(T y)(t)= & \lambda \int_{0}^{1} G(t, s) \omega(s) f(s, y(s)) d s \\
& +\lambda \sum_{k=1}^{m} G\left(t, t_{k}\right) I_{k}\left(t_{k}, y\left(t_{k}\right)\right) \\
\geq & \frac{1}{\sqrt{\mathbf{M}} \sinh (\sqrt{\mathbf{M}})} \lambda \\
& \times\left[\int_{0}^{1} \omega(s) f(s, y(s)) d s+\sum_{k=1}^{m} I_{k}\left(t_{k}, y\left(t_{k}\right)\right)\right] \\
\geq & \delta \frac{\cosh ^{2}(\sqrt{\mathbf{M}})}{\sqrt{\mathbf{M}} \sinh (\sqrt{\mathbf{M}})} \lambda \\
& \times\left[\int_{0}^{1} \omega(s) f(s, y(s)) d s+\sum_{k=1}^{m} I_{k}\left(t_{k}, y\left(t_{k}\right)\right)\right] \\
\geq & \delta\|T y\| .
\end{aligned}
$$

Thus, $T(K) \subset K$.

Next, by similar arguments of Lemmas 5 and 6 [12] one can prove that $T: K \rightarrow K$ is completely continuous. So it is omitted, and the theorem is proved.

To obtain positive solutions of problem (1), the following fixed point theorem in cones is fundamental, which can be found in [23, page 94].

Lemma 7. Let $P$ be a cone in a real Banach space E. Assume $\Omega_{1}, \Omega_{2}$ are bounded open sets in $E$ with $0 \in \Omega_{1}, \bar{\Omega}_{1} \subset \Omega_{2}$. If

$$
A: P \cap\left(\bar{\Omega}_{2} \backslash \Omega_{1}\right) \longrightarrow P
$$

is completely continuous such that either

(a) $\|A x\| \leq\|x\|, \forall x \in P \cap \partial \Omega_{1}$, and $\|A x\| \geq\|x\|, \forall x \in$ $P \cap \partial \Omega_{2}$, or

(b) $\|A x\| \geq\|x\|, \forall x \in P \cap \partial \Omega_{1}$, and $\|A x\| \leq\|x\|, \forall x \in$ $P \cap \partial \Omega_{2}$,

then $A$ has at least one fixed point in $P \cap\left(\bar{\Omega}_{2} \backslash \Omega_{1}\right)$.

\section{Proofs of the Main Results}

For convenience we introduce some notations

$$
\begin{gathered}
\gamma=\int_{0}^{1} \omega(s) d s, \quad \rho=\max _{t \in J, 0 \leq y \leq c} f(t, y), \\
\rho_{*}=\max \left\{\rho_{k}, k=1,2, \ldots, m\right\}, \\
\text { where } \rho_{k}=\max _{t \in J, 0 \leq y \leq c} I_{k}(t, y), k=1,2, \ldots, m \text {, and } c>0 \text { is }
\end{gathered}
$$
a constant.

Proof of Theorem 1. We need only prove this theorem under condition $\left(\mathrm{H}_{3}\right)$ since the proof is similar when $\left(\mathrm{H}_{4}\right)$ holds, provided the proper adjustments are made.

If $f^{0}=0, I^{0}(k)=0$, then there exist $l>0$ and $r>0$ such that

$$
\begin{aligned}
& f(t, y)<l y, \quad I_{k}(t, y)<l y \\
& \forall t \in J, \quad 0 \leq y \leq r, \quad k=1,2, \ldots, m
\end{aligned}
$$

where $l$ satisfies

$$
\lambda \max \{\beta, 1\} l(\gamma+m) \leq 1
$$

Then for $y \in K \cap \partial \Omega_{r}$ we have

$$
\begin{aligned}
\left(T_{\lambda} y\right)(t)= & \lambda \int_{0}^{1} G(t, s) \omega(s) f(s, y(s)) d s \\
& +\lambda \sum_{k=1}^{m} G\left(t, t_{k}\right) I_{k}\left(t_{k}, y\left(t_{k}\right)\right) \\
\leq & \lambda \beta \int_{0}^{1} \omega(s) l y(s) d s+\lambda \beta \sum_{k=1}^{m} l y\left(t_{k}\right) \\
\leq & \lambda \beta l\|y\|_{\mathrm{PC}^{1}}\left(\int_{0}^{1} \omega(s) d s+m\right) \\
= & \lambda \beta l\|y\|_{\mathrm{PC}^{1}}(\gamma+m) \leq\|y\|_{\mathrm{PC}^{1}},
\end{aligned}
$$




$$
\begin{aligned}
\left|\left(T_{\lambda} y\right)^{\prime}(t)\right| \leq & \lambda \int_{0}^{1}\left|G_{t}^{\prime}(t, s)\right| \omega(s) f(s, y(s)) d s \\
& +\lambda \sum_{k=1}^{m}\left|G_{t}^{\prime}\left(t, t_{k}\right)\right| I_{k}\left(t_{k}, y\left(t_{k}\right)\right) \\
\leq & \lambda \int_{0}^{1} \omega(s) f(s, y(s)) d s \\
& +\lambda \sum_{k=1}^{m} I_{k}\left(t_{k}, y\left(t_{k}\right)\right) \\
\leq & \lambda l(\gamma+m)\|y\|_{\mathrm{PC}^{1}} \\
\leq & \|y\|_{\mathrm{PC}},
\end{aligned}
$$

where

$$
\begin{aligned}
& G_{t}^{\prime}(t, s) \\
& = \begin{cases}\frac{-\sinh (\sqrt{\mathbf{M}}(1-t)) \cosh (\sqrt{\mathbf{M}} s)}{\sinh (\sqrt{\mathbf{M}})}, & 0 \leq s \leq t \leq 1, \\
\frac{\cosh (\sqrt{\mathbf{M}} t) \sinh (\sqrt{\mathbf{M}}(1-s))}{\sinh (\sqrt{\mathbf{M}})}, & 0 \leq t \leq s \leq 1, \\
\max _{t, s \in J, t \neq s}\left|G_{1 t}^{\prime}(t, s)\right|=1 . & \end{cases}
\end{aligned}
$$

It follows from (17) that

$$
\left\|T_{\lambda} y\right\|_{\mathrm{PC}^{1}} \leq\|y\|_{\mathrm{PC}^{1}}, \quad \forall y \in K \cap \partial \Omega_{r} .
$$

If $f_{\infty}=\infty, I_{\infty}(k)=\infty$, then there exist $L>0$ and $R>r>0$ such that

$$
\begin{aligned}
& f(t, y)>L y, \quad I_{k}(t, y)>L y, \\
& \forall t \in J, \quad y \geq R, \quad k=1,2, \ldots, m,
\end{aligned}
$$

where $L$ satisfies

$$
\lambda \alpha L \delta(\gamma+m) \geq 1 .
$$

Let $\eta=R / \delta$. Thus, when $y \in K \cap \partial \Omega_{\eta}$ we have

$$
y(t) \geq \delta\|y\|_{\mathrm{PC}^{1}}=\delta \eta=R, \quad t \in J,
$$

and then we get

$$
\begin{aligned}
\left(T_{\lambda} y\right)(t)= & \lambda \int_{0}^{1} G(t, s) \omega(s) f(s, y(s)) d s \\
& +\lambda \sum_{k=1}^{m} G\left(t, t_{k}\right) I_{k}\left(t_{k}, y\left(t_{k}\right)\right) \\
\geq & \lambda \alpha \int_{0}^{1} \omega(s) L y(s) d s+\lambda \alpha \sum_{k=1}^{m} L y\left(t_{k}\right) \\
\geq & \lambda \alpha L \delta\|y\|_{\mathrm{PC}^{1}}\left(\int_{0}^{1} \omega(s) d s+m\right) \\
= & \lambda \alpha L \delta\|y\|_{\mathrm{PC}^{1}}(\gamma+m) \\
\geq & \|y\|_{\mathrm{PC}^{1}} .
\end{aligned}
$$

This yields

$$
\left\|T_{\lambda} y\right\|_{\mathrm{PC}^{1}} \leq\|y\|_{\mathrm{PC}^{1}}, \quad \forall y \in K \cap \partial \Omega_{\eta}
$$

Hence, for given $\lambda>0$ condition (a) of Lemma 7 is satisfied of operator $T_{\lambda}$, which implies that $T_{\lambda}$ has a fixed point $y_{\lambda}$ in $\bar{\Omega}_{\eta} \backslash \Omega_{r}$.

It remains to prove $\left\|y_{\lambda}\right\|_{\mathrm{PC}^{1}}=+\infty$ as $\lambda \rightarrow 0^{+}$. In fact, if not, there would exist a number $c>0$ and a sequence $\lambda_{n} \rightarrow$ $0^{+}$such that

$$
\left\|y_{\lambda_{n}}\right\|_{\mathrm{PC}^{1}} \leq c \quad(n=1,2,3, \ldots) .
$$

Furthermore, the sequence $\left\{\left\|y_{\lambda_{n}}\right\|_{\mathrm{PC}^{1}}\right\}$ contains a subsequence that converges into a number $d$, where $0 \leq d \leq c$. For simplicity, suppose that $\left\{\left\|y_{\lambda_{n}}\right\|_{\mathrm{PC}^{1}}\right\}$ itself converges into $d$.

If $d>0$, then $\left\|y_{\lambda_{n}}\right\|_{\mathrm{PC}^{1}}>d / 2$ for sufficiently large $n(n>$ $\mathbb{N})$, and therefore

$$
\begin{aligned}
\frac{1}{\lambda_{n}}= & \left(\| \int_{0}^{1} G(t, s) \omega(s) f\left(s, y_{\lambda_{n}}(s)\right) d s\right. \\
& \left.\quad+\sum_{k=1}^{m} G\left(t, t_{k}\right) I_{k}\left(t_{k}, y_{\lambda_{n}}\left(t_{k}\right)\right) \|_{\mathrm{PC}^{1}}\right) \\
& \times\left(\left\|y_{\lambda_{n}}\right\|_{\mathrm{PC}^{1}}\right)^{-1} \\
\leq & \frac{\beta\left(\gamma \rho+m \rho^{*}\right)}{\left\|y_{\lambda_{n}}\right\|_{\mathrm{PC}^{1}}} \\
\leq & \frac{2 \beta\left(\gamma \rho+m \rho^{*}\right)}{d} \quad(n>\mathbb{N}),
\end{aligned}
$$

which contradicts $\lambda_{n} \rightarrow 0^{+}$.

If $d=0$, then $\left\|y_{\lambda_{n}}\right\|_{\mathrm{PC}^{1}} \rightarrow 0$ for sufficiently large $n$ ( $n>$ $\mathbb{N})$, and therefore it follows from $\left(\mathrm{H}_{3}\right)$ that for any $\varepsilon>0$ there exists $b>0$ such that

$$
\begin{aligned}
f\left(t, y_{\lambda_{n}}(t)\right) \leq \varepsilon y_{\lambda_{n}}, \quad & I_{k}\left(t, y_{\lambda_{n}}(t)\right) \leq \varepsilon y_{\lambda_{n}}, \\
& \forall y_{\lambda_{n}}: 0 \leq y_{\lambda_{n}} \leq b,
\end{aligned}
$$

and hence it follows from (10) that

$$
\begin{aligned}
\frac{1}{\lambda_{n}}= & \left(\| \int_{0}^{1} G(t, s) \omega(s) f\left(s, y_{\lambda_{n}}(s)\right) d s\right. \\
& \left.\quad+\sum_{k=1}^{m} G\left(t, t_{k}\right) I_{k}\left(t_{k}, y_{\lambda_{n}}\left(t_{k}\right)\right) \|_{\mathrm{PC}^{1}}\right) \\
& \times\left(\left\|y_{\lambda_{n}}\right\|_{\mathrm{PC}^{1}}\right)^{-1} \\
\leq & \frac{\beta\left(\gamma \varepsilon\left\|y_{\lambda_{n}}\right\|_{\mathrm{PC}^{1}}+m \varepsilon\left\|y_{\lambda_{n}}\right\|_{\mathrm{PC}^{1}}\right)}{\left\|y_{\lambda_{n}}\right\|_{\mathrm{PC}^{1}}} \\
= & \beta(\gamma+m) \varepsilon .
\end{aligned}
$$

Since $\varepsilon$ is arbitrary, we have $\lambda_{n} \rightarrow+\infty \quad(n \rightarrow+\infty)$ in contradiction to $\lambda_{n} \rightarrow 0^{+}$. Therefore, $\left\|y_{\lambda}\right\| \rightarrow+\infty$ as $\lambda \rightarrow 0^{+}$and the proof is complete. 
Remark 8. Comparing with Nieto and O'Regan [17], the main features of this paper are as follows.

(i) The parameter $\lambda>0$ is considered.

(ii) The parameter dependence of the solution is available.

(iii) $\omega \in L_{\mathrm{loc}}^{1}(0,1)$, not $\omega(t) \equiv 1$ for $t \in J$.

\section{An Example}

To illustrate how our main results can be used in practice we present an example.

Example 9. Consider the following boundary value problems

$$
\begin{gathered}
-y^{\prime \prime}(t)+y(t)=\lambda \frac{1}{\sqrt{t}} \sqrt[3]{1+t^{2}} y^{2}(t), \quad t \in J, \quad t \neq \frac{1}{2}, \\
-\left.\Delta y^{\prime}\right|_{t_{1}=1 / 2}=\frac{1}{1+t} \frac{y^{3}}{1+y}, \quad k=1, \\
y^{\prime}(0)=y^{\prime}(1)=0 .
\end{gathered}
$$

Evidently, $y(t) \equiv 0$ is the trivial solution of problem (29).

Conclusion. Problem (29) has at least one positive solution for any $\lambda>0$.

Proof. Problem (29) can be regarded as a problem of the form (1), where

$$
\begin{gathered}
\mathbf{M}=1, \quad t_{1}=\frac{1}{2} \\
\omega(t)=\frac{1}{\sqrt{t}}, \quad f(t, y)=\sqrt[3]{1+t^{2}} y^{2}(t), \\
I_{1}(t, y)=(1+t) \frac{y^{3}}{1+y} .
\end{gathered}
$$

It follows from the definition of $\omega, f$, and $I$ that $\left(\mathrm{H}_{1}\right)$ and $\left(\mathrm{H}_{2}\right)$ hold, and $\omega(t)$ is singular at $t=0$ and $t=1$. By calculating, we have

$$
\begin{gathered}
\delta=\frac{1}{\cosh ^{2}(1)}, \quad \gamma=\int_{0}^{1} \frac{1}{\sqrt{t}} d t=2, \\
\alpha=\frac{1}{\sinh (1)}, \quad \beta=\frac{\cosh ^{2}(1)}{\sinh (1)}, \\
f^{0}=\limsup _{y \rightarrow 0} \max _{t \in J} \frac{\sqrt[3]{1+t^{2}} y^{2}(t)}{y}=0, \\
I^{0}(k)=\limsup _{y \rightarrow 0} \max _{t \in J} \frac{(1+t)\left(y^{3} /(1+y)\right)}{y}=0, \\
f_{\infty}=\liminf _{y \rightarrow \infty} \min _{t \in J} \frac{\sqrt[3]{1+t^{2}} y^{2}(t)}{y}=\infty \\
I_{\infty}(k)=\limsup _{y \rightarrow \infty} \min _{t \in J} \frac{(1+t)\left(y^{3} /(1+y)\right)}{y}=\infty .
\end{gathered}
$$

Then, the condition $\left(\mathrm{H}_{3}\right)$ of Theorem 1 holds. Hence, by Theorem 1, the conclusion follows, and the proof is complete.

\section{Conflict of Interests}

The author declares that there is no conflict of interests regarding the publication of this paper.

\section{Acknowledgments}

The authors are indebted to the referee's suggestions. These have greatly improved this paper. This work is sponsored by the Project NSFC (11301178) and the Fundamental Research Funds for the Central Universities (2014MS58).

\section{References}

[1] M. Benchohra, J. Henderson, and S. Ntouyas, Impulsive Differential Equations and Inclusions, Contemporary Mathematics and Its Applications, Hindawi Publishing Corporation, New York, NY, USA, 2006.

[2] A. M. Samoilenko and N. A. Perestyuk, Impulsive Differential Equations, World Scientific Publishing, River Edge, NJ, USA, 1995.

[3] V. Lakshmikantham, D. D. Baĭnov, and P. S. Simeonov, Theory of Impulsive Differential Equations, vol. 6 of Series in Modern Applied Mathematics, World Scientific, Teaneck, NJ, USA, 1989.

[4] S. Gao, L. Chen, J. J. Nieto, and A. Torres, "Analysis of a delayed epidemic model with pulse vaccination and saturation incidence," Vaccine, vol. 24, pp. 6037-6045, 2006.

[5] A. F. B. A. Prado, "Bi-impulsive control to build a satellite constellation," Nonlinear Dynamics and Systems Theory, vol. 5, no. 2, pp. 169-175, 2005.

[6] W. Li, Q. Li, X. Liu, and F. Cui, "Principal and nonprincipal solutions of impulsive dynamic equations with applications," The Journal of Applied Analysis and Computation, vol. 2, no. 4, pp. 431-440, 2012.

[7] Y. Liu and D. O’Regan, "Multiplicity results using bifurcation techniques for a class of boundary value problems of impulsive differential equations," Communications in Nonlinear Science and Numerical Simulation, vol. 16, no. 4, pp. 1769-1775, 2011.

[8] R. Ma, B. Yang, and Z. Wang, "Positive periodic solutions of first-order delay differential equations with impulses," Applied Mathematics and Computation, vol. 219, no. 11, pp. 6074-6083, 2013.

[9] X. Hao, L. Liu, and Y. Wu, "Positive solutions for second order impulsive differential equations with integral boundary conditions," Communications in Nonlinear Science and Numerical Simulation, vol. 16, no. 1, pp. 101-111, 2011.

[10] M. Feng and D. Xie, "Multiple positive solutions of multipoint boundary value problem for second-order impulsive differential equations," Journal of Computational and Applied Mathematics, vol. 223, no. 1, pp. 438-448, 2009.

[11] X. Zhang, M. Feng, and W. Ge, "Existence of solutions of boundary value problems with integral boundary conditions for second-order impulsive integro-differential equations in Banach spaces," Journal of Computational and Applied Mathematics, vol. 233, no. 8, pp. 1915-1926, 2010. 
[12] J. Yan, "Existence of positive periodic solutions of impulsive functional differential equations with two parameters," Journal of Mathematical Analysis and Applications, vol. 327, no. 2, pp. 854-868, 2007.

[13] X. Liu and D. Guo, "Method of upper and lower solutions for second-order impulsive integro-differential equations in a Banach space," Computers \& Mathematics with Applications, vol. 38, no. 3-4, pp. 213-223, 1999.

[14] D. Guo, "Extremal solutions for $n$ th-order impulsive integrodifferential equations on the half-line in Banach spaces," Nonlinear Analysis. Theory, Methods \& Applications A, vol. 65, no. 3, pp. 677-696, 2006.

[15] D. Guo, "Variational approach to a class of impulsive differential equations," Boundary Value Problems, vol. 2014, article 37, 2014.

[16] J. Zhou and Y. Li, "Existence and multiplicity of solutions for some Dirichlet problems with impulsive effects," Nonlinear Analysis. Theory, Methods \& Applications A, vol. 71, no. 7-8, pp. 2856-2865, 2009.

[17] J. J. Nieto and D. O’Regan, "Variational approach to impulsive differential equations," Nonlinear Analysis. Real World Applications, vol. 10, no. 2, pp. 680-690, 2009.

[18] J. Xiao, J. J. Nieto, and Z. Luo, "Multiplicity of solutions for nonlinear second order impulsive differential equations with linear derivative dependence via variational methods," Communications in Nonlinear Science and Numerical Simulation, vol. 17, no. 1, pp. 426-432, 2012.

[19] Y. Tian and W. Ge, "Variational methods to Sturm-Liouville boundary value problem for impulsive differential equations," Nonlinear Analysis. Theory, Methods \& Applications A, vol. 72, no. 1, pp. 277-287, 2010.

[20] M. Feng, X. Li, and C. Xue, "Multiple positive solutions for impulsive singular boundary value problems with integral boundary conditions," International Journal of Open Problems in Computer Science and Mathematics, vol. 2, no. 4, pp. 546-561, 2009.

[21] J. Sun, H. Chen, and L. Yang, "The existence and multiplicity of solutions for an impulsive differential equation with two parameters via a variational method," Nonlinear Analysis. Theory, Methods \& Applications A, vol. 73, no. 2, pp. 440-449, 2010.

[22] P. Ning, Q. Huan, and W. Ding, "Existence result for impulsive differential equations with integral boundary conditions," Abstract and Applied Analysis, vol. 2013, Article ID 134691, 9 pages, 2013.

[23] D. J. Guo and V. Lakshmikantham, Nonlinear Problems in Abstract Cones, Academic Press, NewYork, NY, USA, 1988.

[24] X. Liu and Y. Li, "Positive solutions for Neumann boundary value problems of second-order impulsive differential equations in Banach spaces," Abstract and Applied Analysis, vol. 2012, Article ID 401923, 14 pages, 2012. 


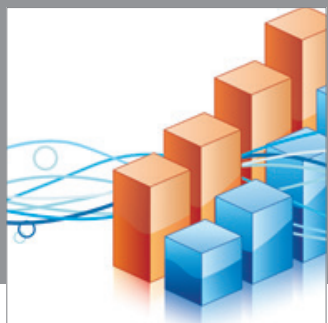

Advances in

Operations Research

mansans

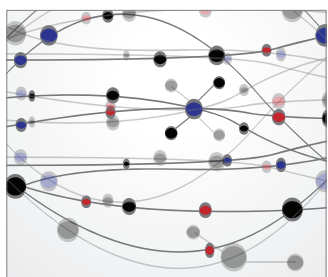

The Scientific World Journal
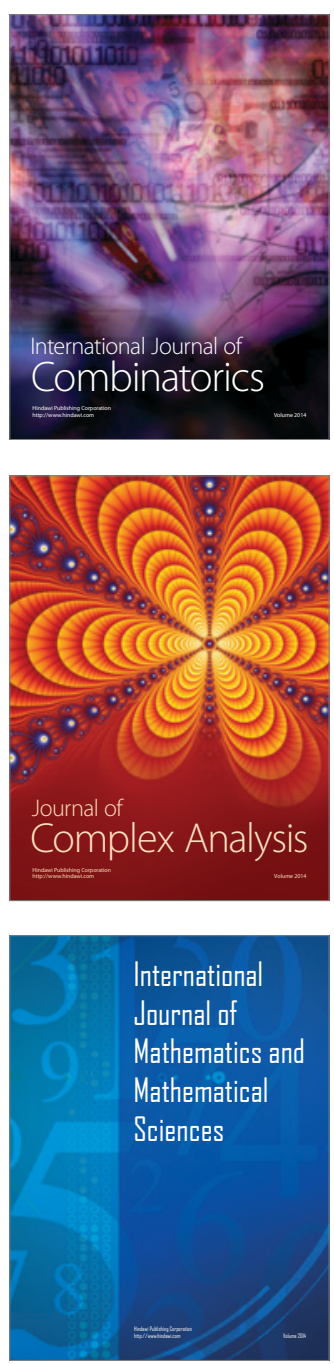
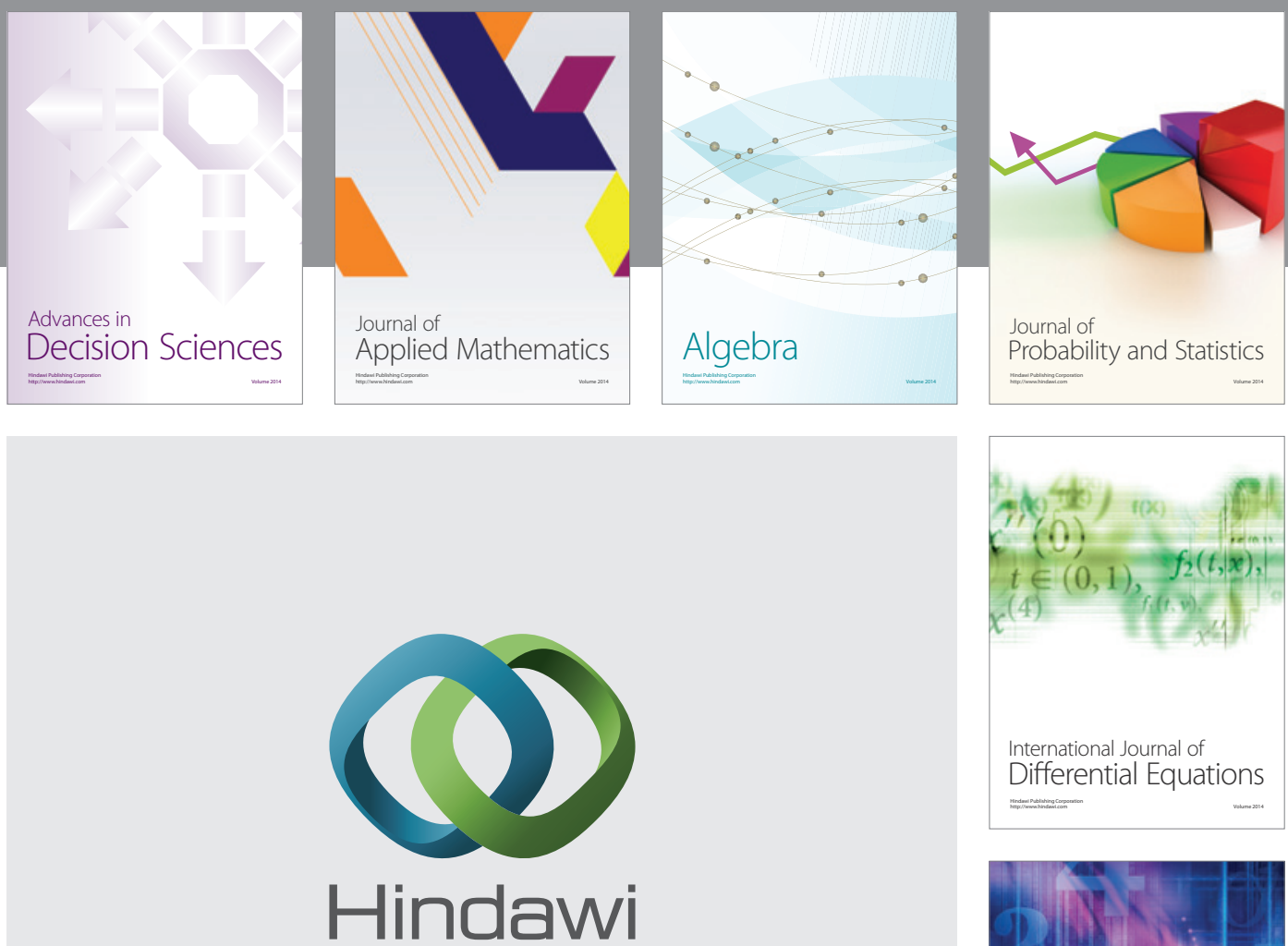

Submit your manuscripts at http://www.hindawi.com
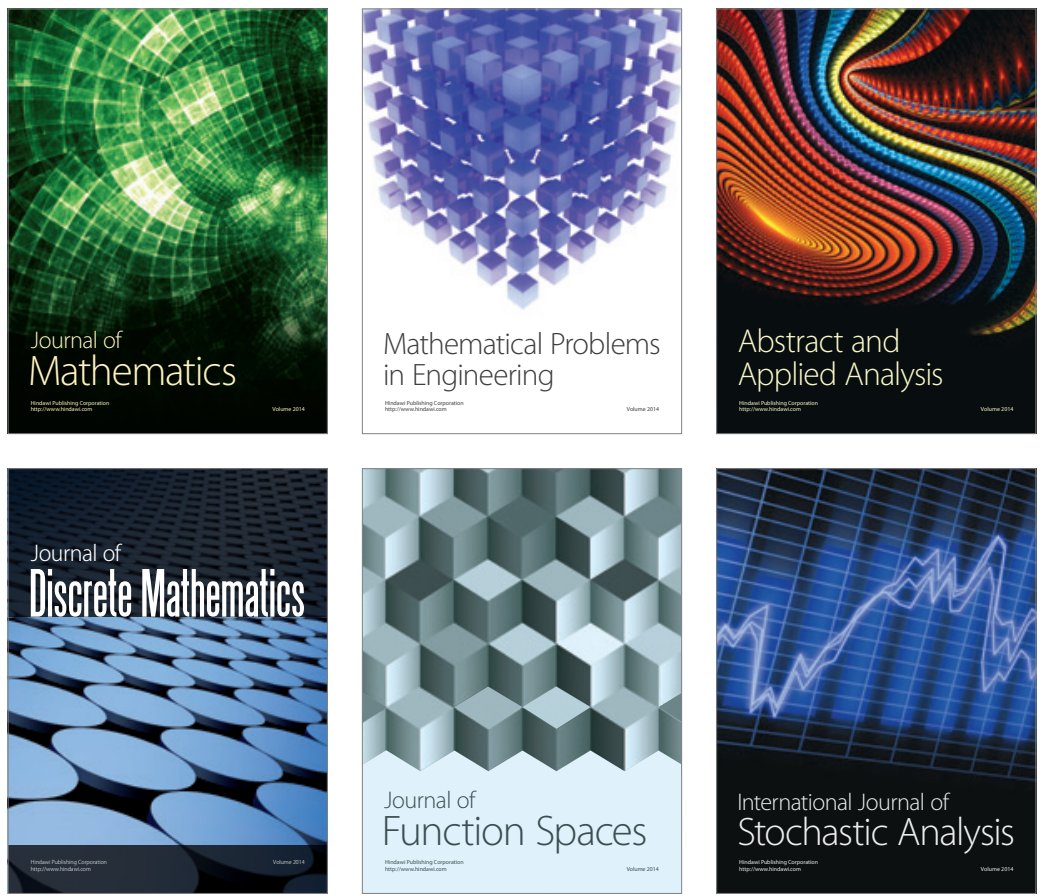

Journal of

Function Spaces

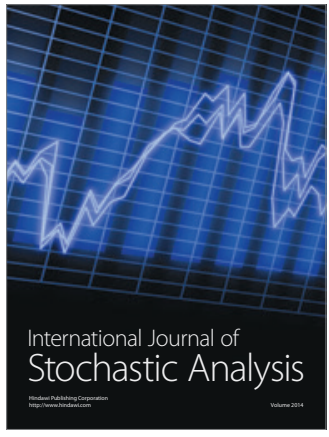

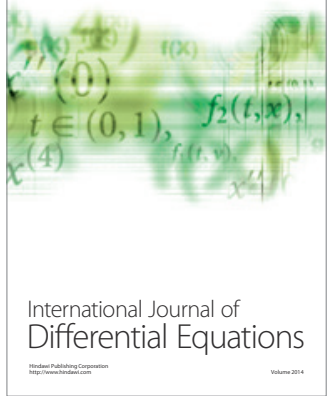
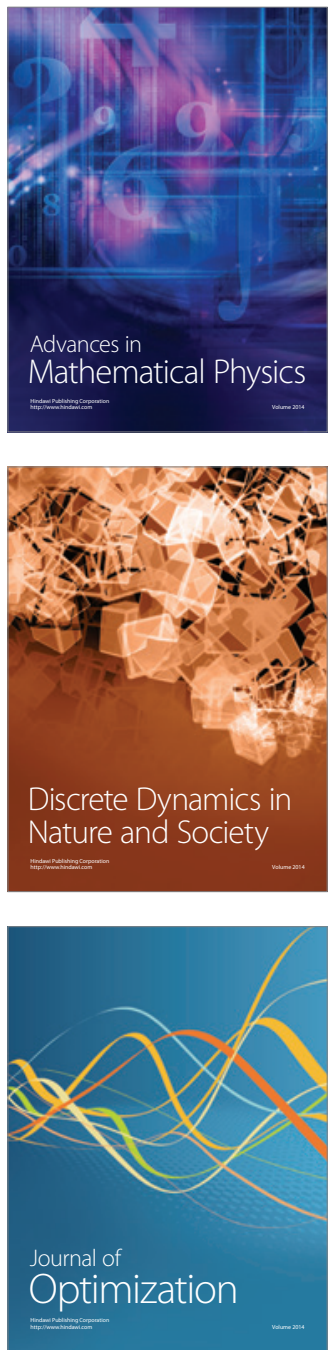